\title{
EXISTENCE OF POLYMORPHIC GENOTYPIC EQUILIBRIA UNDER ASYMMETRY OF HOMOZYGOTIC DISADVANTAGE AND A DEFICIENCY OF HETEROZYGOTES
}

\author{
MARTIN ZIEHE \\ Abteilung Forstgenetik und Forstpflanzenzüchtung der Universität Göttingen, D-3400 \\ Göttingen-Weende, Büsgenweg 2, Federal Republic of Germany
}

Received 11.vii.83

SUMMARY

\begin{abstract}
A general relationship is derived, which necessarily holds in polymorphic overdominance equilibria. Selective asymmetry in homozygotic fitnesses as well as heterozygotic frequency deficiency produced by mating system effects are both involved in this relationship. The measurement of the asymmetry in homozygotic disadvantage is based on effective gametic contributions to the offspring generation (Darwinian fitness) and may also reflect selective effects via reproduction. The heterozygotic deficiency is measured relative to Hardy-Weinberg proportions and is regarded as a consequence of particular mating system components. The relationship implies that, by simultaneously increasing asymmetry and heterozygotic deficiency, the exclusion of polymorphic equilibria becomes more likely, or, equivalently, that a certain closeness to symmetry in homozygotic disadvantage along with a sufficient number of heterozygotes is necessary to admit them. The result is applied to a multiallelic model of differential pollen production combined with partial self-fertilisation as well as to a model of genotypically different rates of self-fertilisation or positive assortative mating.
\end{abstract}

\section{INTRODUCTION}

The maintenance of selectively balanced genetic polymorphisms under those non-random mating systems which lead to a deficiency of heterozygotes presents a fundamental problem in population genetics. It is the object of numerous experimental and theoretical considerations, most of which refer to partial self-fertilisation as the underlying mating system effect. $A$ basic example concerns the classical diallelic viability selection model. If, for a plant population with viability overdominance, instead of completely random gametic fusion partial self-fertilisation occurs, the conditions for the existence of a polymorphic equilibrium and protectedness of the polymorphism are drastically changed. It is well known that overdominance in viabilities is no longer sufficient to guarantee the maintenance of the polymorphism if partial self-fertilisation is introduced. Based on their detailed analytical considerations with respect to this model, Kimura and Ohta (1971) suggested that "overdominance cannot be a major mechanism for polymorphism in predominantly self-fertilising organisms, unless the overdominance is very strong". Several years later, Brown (1979) took up Kimura and Ohta's basic inequality and pointed out that a further effect in addition to the rate of self-fertilisation greatly influences the existence condition for a polymorphic equilibrium. He described this effect as "the degree of asymmetry" between $s_{1}$ and $s_{2}$, the "selection coefficients against the homozygotes". This statement is impressively confirmed by Hayman's (1953) phase 
diagrams. Ziehe (1982) presented similar results for models in which particular selection effects occur during reproduction (e.g., differential pollen production) under mixed random mating and selfing. This raises a more general question: Suppose selection (in viability or during reproduction) is combined with a non-random mating system inducing a heterozygote deficiency (such as partial self-fertilisation or positive assortative mating). What kind of relationship between heterozygote deficiency and asymmetry in homozygotic disadvantage must hold to allow for the existence of a polymorphic equilibrium? To investigate this, the selection effect realised after survival and during reproduction must be measured in terms of Darwinian fitness, which reflect the total amount of genetic (allelic) contributions individuals are able to incorporate into their zygotic offspring.

The final aim of this paper is:

(i) to answer the above question for a model with one gene locus and two alleles,

(ii) to present a multiallelic extension,

(iii) to demonstrate and illustrate the results for two examples of models with selection acting via reproduction.

\section{Basic Assumptions and Notations}

A diploid organism reproducing with discrete generations is considered within a population of effectively infinite size. The subsequent investigations deal with a single multiallelic gene locus with alleles $A_{1}, A_{2}, \ldots$ and (unordered) genotypes $A_{1} A_{1}, A_{1} A_{2}, A_{2} A_{2}, \ldots p_{i}$ denotes the relative frequency of the allele $A_{i}$, and $P_{i j}$ that of the genotype $A_{i} A_{j}$, both being measured at the zygotic population stage.

The Darwinian fitness $w_{i j}$ of a genotype $A_{i} A_{j}$ is defined as the mean number of successful gametes that individuals (at zygotic census) of this genotype are able to contribute on the average to offspring zygotes (compare e.g., Gregorius and Ross, 1981). These $w_{i j}$ 's measure directly the extent to which genotypes transmit their genes to the subsequent generation and thus include survival abilities as well as reproductive efficiencies. Here $w_{i j}$ may depend on the actual genotypic structure: $w_{i j}=w_{i j}(P)$ (examples later in this paper). The term " $(P)$ " denotes the functional dependence.

The following basic assumption which is assumed to hold throughout this paper, reflects the effect of Mendelian segregation with respect to fitness: An adult heterozygote $A_{i} A_{j}$ produces equal amounts of successful gametes containing $A_{i}$ and $A_{j}$. As above, a gamete is called successful if it is incorporated into a zygotic offspring.

Then the mean allelic fitness $w_{i}$ of an allele $A_{i}$ can be written as

$$
w_{i}=w_{i}(P)=\left(P_{i i} / p_{i}\right) w_{i i}+\frac{1}{2} \sum_{j, j \neq i}\left(P_{i j} / p_{i}\right) w_{i j}
$$

and the change in allelic frequencies from one generation to the next occurs according to (e.g., Gregorius, 1981)

$$
p_{i}^{\prime}=p_{i} w_{i}(P) /\left(\sum_{j} p_{j} w_{j}(P)\right)
$$

Primes indicate frequencies in the offspring generation. 


\section{The Diallelic Case}

\section{(i) Pre-requisites}

The basic effects under consideration will be quantified as follows.

A deficiency of heterozygotes relative to Hardy-Weinberg proportions can be measured by the panmictic index $I$,

$$
I=I(P)=P_{12} / 2 p_{1} p_{2} \text {. }
$$

Equivalently, Wright's fixation index $F(P), F=1-I$, can be used. Both parameters are frequently used to quantify inbreeding effects on genotypic structures (e.g., Jain and Workman, 1967). $I=1$ clearly represents HardyWeinberg proportions, while a decreasing $I$ reflects an increasing deficiency of heterozygotes.

A parameter $\gamma$ measures the asymmetry of deviations of homozygotic from heterozygotic fitness:

$$
\gamma=\gamma(P)=\left(w_{12}-w_{22}\right) /\left(w_{12}-w_{11}\right) .
$$

This parameter has proved to be useful in characterising allelic constancy or equilibrium locations for several population genetic models (e.g., Gregorius, 1982 or Ziehe, 1982). Some properties of $\gamma$ are listed in table 1.

TABLE 1

Schematic diagram of $I$ and $\gamma$ as measures of heterozygote deficiency and allelic selective

\begin{tabular}{|c|c|c|c|}
\hline Parameter & 1 & $\stackrel{\text { Parameter range }}{\longrightarrow}$ & 0 \\
\hline & & Increasing & \\
\hline $\begin{array}{c}I \\
(F=1-I)\end{array}$ & $\begin{array}{c}\text { Hardy-Weinberg } \\
\text { proportions } \\
P_{12}=2 p_{1} p_{2}\end{array}$ & $\begin{array}{l}\text { heterozygote } \\
\text { deficiency }\end{array}$ & $\begin{array}{l}\text { No heterozygotes } \\
\qquad P_{12}=0\end{array}$ \\
\hline$\gamma$ & Allelic selective & $\begin{array}{l}\text { Increasing } w_{22}: \\
\text { Increasing }\end{array}$ & \\
\hline $\begin{array}{c}\text { (provided } \\
\left.w_{11} \leq w_{22} \leq w_{12}\right)\end{array}$ & $\begin{array}{c}\text { symmetry } \\
w_{11}=w_{22}<w_{12}\end{array}$ & $\overrightarrow{\text { asymmetry }}$ & $\begin{array}{l}\text { Complete dominance } \\
\qquad w_{11}<w_{22}=w_{12}\end{array}$ \\
\hline
\end{tabular}
asymmetry respectively

\section{(ii) Results}

Now we assume the existence of a polymorphic equilibrium $\hat{P}$ with overdominance in fitness $\left(\hat{w}_{11}, \hat{w}_{22}<\hat{w}_{12}\right)$. By suitable numbering of the alleles, without loss of generality, $\hat{w}_{11} \leq \hat{w}_{22}$. Here "A "' indicates parameters and frequencies in equilibrium $P$. Allelic constancy necessarily holds for a genotypic equilibrium and according to (1) implies identical mean allelic fitnesses $\left(\hat{w}_{1}=\hat{w}_{2}\right)$. In the diallelic case, $\hat{w}_{i}$ reduces to

$$
\hat{w}_{i}=\hat{P}_{i i} \hat{w}_{i i} / \hat{p}_{i}+\frac{1}{2} \hat{P}_{12} \hat{w}_{12} / \hat{p}_{i} \text {. }
$$

Since $\hat{w}_{22} \leqq \hat{w}_{2} \leqq \hat{w}_{12}$ always holds, the allelic constancy in particular requires $\hat{w}_{22} \leq \hat{w}_{1}$. This is equivalent to

$$
1 \leqq \hat{\gamma}+\hat{p}_{2} \hat{I} .
$$


Thus ignoring the trivial case $\hat{I}=0$ (no heterozygotes produced, identical homozygotic fitnesses), the inequality

$$
1<\hat{\gamma}+\hat{I} \quad \text { (or, equivalently, } \hat{F}<\hat{\gamma} \text { ) }
$$

is a necessary condition for the polymorphic equilibrium $\hat{\boldsymbol{P}}$ representing fitnesses $\hat{w}_{11} \leqq \hat{w}_{22}<\hat{w}_{12}$. Hence, the deficiency of heterozygotes and the allelic selective asymmetry as realised simultaneously in a polymorphic overdominance equilibrium are necessarily restricted by the requirement that the sum of $\hat{\gamma}$ and $\hat{I}$ must exceed 1 .

$\mathrm{Li}$ (1955) arrived at an inequality necessary for the existence of a polymorphic overdominance equilibrium which analytically corresponds to inequality (2), if the parameter $\gamma$ is written in terms of viabilities. If no selection occurs during reproduction, Li's inequality and inequality (2) indeed must coincide. However, inequality (2) as a necessary condition within the polymorphic equilibrium is valid in a more general context if a $\gamma$ is used which refers to fitnesses in the Darwinian sense. In a later section inequality (2) will be applied to models with selection via reproduction: (i) Selection in pollen production combined with partial self-fertilisation and (ii) genotypically differential partial self-fertilisation or positive assortative mating.

Inequality (2) allows for a relatively simple interpretation of the cumulative impact of both underlying effects on polymorphic equilibrium existence. Suppose that inbreeding effects via particular mating system components necessarily restrict the amount of heterozygotes which would have to be present in hypothetical polymorphic equilibrium, such that $\hat{I}$ falls substantially short of 1 . Suppose further that the asymmetry in allele effects must be realized to such an extent that $\gamma$ is also sufficiently less than 1 . The validity of the reverse direction of inequality (2) leads to a contradiction to the assumed existence of a corresponding polymorphic overdominance equilibrium. Then, however, no such equilibrium can exist.

The examples presented later prove that inequality (2), when applied to particular models, may even deliver necessary and sufficient parameter conditions for the existence of polymorphic equilibria.

\section{A Multiallelic Extension}

(i) Pre-requisites

Now, $n$ alleles $A_{1}, \ldots, A_{n}$ are considered with respect to their coexistence within a polymorphic equilibrium $\hat{P}$. To allow for considerations similar to the diallelic case and avoid an unsurveyable amount of parameters, a restriction concerning genotypic fitness identities within $\hat{P}$ is proposed (see also table 2): All heterozygotes show identical fitness behaviour $\hat{w}_{\text {het }}$, homozygotes are divided into one group consisting of $m$ homozygotes with fitness $\hat{w}_{a}$ and another consisting of $r$ homozygotes with homozygotic fitness $\hat{w}_{b}(m+r=n)$, where, without loss of generality, $\hat{w}_{a} \leq \hat{w}_{b}$.

The multiallelic version of heterozygotic advantage within $\hat{P}$ ( $\hat{w}_{\text {het }} \geqq$ $\hat{w}_{a}, \hat{w}_{b}$ ) will be investigated. Using this, table 2 corresponds to a homozygosity effect realised at two different levels and a constant heterosis not depending on the particular alleles involved in heterozygotes. 
TABLE 2

Genotypic fitnesses within a polymorphic equilibrium $\hat{P}$

\begin{tabular}{cc}
\hline Genotypes & Genotypic fitness \\
\hline Heterozygotes & \\
$A_{1} A_{2}, \ldots, A_{n-1} A_{n}$ & $\hat{w}_{\text {het }}$ \\
$m$ Homozygotes & \\
$A_{1} A_{1}, \ldots, A_{m} A_{m}$ & $\hat{w}_{a}$ \\
$r$ Homozygotes & \\
$A_{m+1} A_{m+1}, \ldots, A_{m+r} A_{m+r}$ & $\hat{w}_{b}$ \\
\hline
\end{tabular}

$\hat{\gamma}$ defined by $\hat{\gamma}=\left(\hat{w}_{\text {het }}-\hat{w}_{b}\right) /\left(\hat{w}_{\text {het }}-\hat{w}_{a}\right)$ again measures the asymmetry in homozygotic fitness disadvantage relative to the heterozygote and thus the asymmetry in allele effects between two alleles taken from the different groups.

Let $I_{i j}$ denote the deficiency of $A_{i} A_{j}$ heterozygotes relative to corresponding Hardy-Weinberg proportions: $I_{i j}(P)=P_{i j} /\left(2 p_{i} p_{j}\right)$. A basic assumption for this section consists in $I_{i j}=\hat{I}$, which means that in $p$ the reduction of the heterozygotic frequency relative to Hardy-Weinberg proportions is identical with respect to all heterozygotic genotypes.

\section{(ii) Results}

The mean allelic fitnesses in equilibrium $\hat{P}$ allow for the following simplification:

$$
\hat{w}_{k}=\hat{w}_{a}+\hat{I}\left(1-\hat{p}_{k}\right)\left(\hat{w}_{\text {het }}-\hat{w}_{a}\right) \text { for } k=1, \ldots, m
$$

and

$$
\hat{w}_{l}=\hat{w}_{b}+\hat{I}\left(1-\hat{p}_{l}\right)\left(\hat{w}_{\text {het }}-\hat{w}_{b}\right) \quad \text { for } l=m+1, \ldots, n .
$$

$\hat{w}_{k}=\hat{w}_{l}$ for arbitrary $k$ and $l$ is a necessary equilibrium condition and is equivalent to $1-\hat{I}\left(1-\hat{p}_{k}\right)=\hat{\gamma}\left[1-\hat{I}\left(1-\hat{p}_{l}\right)\right]$. Clearly, $\hat{I}=0$ requires $\hat{w}_{a}=\hat{w}_{b}$ $(\hat{\gamma}=1)$ and leads to a trivial equilibrium. Let $\hat{I}>0$. Inserting $\hat{p}:=\hat{p}_{k}$ for $k=1, \ldots, m$ and $\hat{p}_{l}=(1-m \hat{p}) / r$ for $l=m+1, \ldots, n$ delivers the solution

$$
\hat{p}=[\hat{I} \hat{\gamma}-r(1-\hat{I})(1-\hat{\gamma})] /[\hat{I}(r+m \hat{\gamma})] \text {. }
$$

$m \hat{p}$ is obviously always less than 1 , but $\hat{p}>0$ requires

$$
\frac{\hat{I}}{1-\hat{I}} \frac{\hat{\gamma}}{1-\hat{\gamma}}>r \text {. }
$$

Thus, inequality (4) is a necessary condition for the polymorphic equilibrium $\hat{P}$ with $\hat{w}_{a} \leq \hat{w}_{b}<\hat{w}_{\text {het }}$. Again, as in the preceding section, the reverse direction of inequality (4) can serve to exclude a priori the existence of polymorphic equilibria.

It should be noted that inequality (4) only depends on the number $r$ of alleles leading to those homozygotes with the higher equilibrium fitness $\hat{w}_{b}$. Inequality (4) is not affected if only the number $m$ (and thus also the total number of alleles) increases. 
For $r=1$, inequality (4) reduces to inequality (2), which is valid for the diallelic case. When the number of those alleles leading to the higher homozygotic fitness increases, the domain for parameters $\hat{I}$ and $\hat{\gamma}$ which allows for the existence of polymorphic equilibria rapidly decreases, while the exclusion domain increases.

The exclusion procedure is efficient if both $\hat{I}$ and $\hat{\gamma}$ deviate to a certain extent from the "ideal" case " 1 " (compare table 1). Supplementing inequality (4), the following equivalent formulations are necessary for equilibrium existence:

$$
\hat{F}=1-\hat{I}<\hat{\gamma} /[\hat{\gamma}+r(1-\hat{\gamma})]
$$

or

$$
1-\hat{\gamma}<\hat{I} /[\hat{I}+r(1-\hat{I})] \text {. }
$$

\section{Applications}

(i) Differential pollen production in partially self-fertilising plant populations

Model features: Plants of genotype $A_{i} A_{i}$ produce on the average a number $\phi$ of ovules, $\sigma \phi$ of which are reserved for and fertilised via selffertilisation (identical for all genotypes); the remaining number of ovules is fertilised at random by pollen from the pollen cloud to which individuals of genotypes $A_{i} A_{j}$ contribute on the average an amount $\mu_{i j}$ of pollen (genotypically differential cross-fertilisation contributions). Let $\sigma \neq 1$.

Allelic selective asymmetry: The mean gametic contribution of individuals with genotype $A_{i} A_{j}$ to offspring zygotes results in:

$$
\begin{array}{ll}
\phi & \text { (gametic contribution via ovules) } \\
+\sigma \phi & \text { (pollen contribution via ovule self-fertilisation) } \\
+(1-\sigma) \phi \mu_{i j} / \bar{\mu} & \begin{array}{l}
\text { (cross-fertilisation pollen contribution incorporated into } \\
\text { zygotes), }
\end{array}
\end{array}
$$

where $\bar{\mu}=\sum_{j, i, i \leqq j} P_{i j} \mu_{i j}$ is the population mean of pollen cloud contributions. Thus

$$
w_{i j}=w_{i j}(P)=(1+\sigma) \phi+(1-\sigma) \phi \mu_{i j} / \bar{\mu},
$$

which is frequency dependent due to pollen competition for fertilisation.

Restricting the multiallelic considerations to $\mu_{11}=\cdots=\mu_{m m}=\mu_{a}$, $\mu_{m+1, m+1}=\cdots=\mu_{m+r, m+r}=\mu_{b}$ and $\mu_{i j}=\mu_{\text {het }}$ for $i \neq j$ with $\mu_{a} \leqq \mu_{b}<\mu_{\text {het }}$, then the required fitness identities hold and

$$
\gamma=\frac{\left(w_{\text {het }}-w_{b}\right)}{\left(w_{\text {het }}-w_{a}\right)}=\frac{\left(\mu_{\text {het }}-\mu_{b}\right)}{\left(\mu_{\text {het }}-\mu_{a}\right)}
$$

turns out to be frequency independent and has the same value for each polymorphic genotypic structure. Thus $\gamma=\hat{\gamma}$.

Heterozygote frequency deficiency in equilibrium: The transition equations read (compare e.g., Gregorius and Ross, 1981)

$$
P_{i j}^{\prime}=\sigma P_{i j} / 2+(1-\sigma) 2 p_{i} p_{i}\left(\mu_{i}(P)+\mu_{j}(P)\right) / \bar{\mu} \quad \text { for } i \neq j,
$$

where

$$
\mu_{i}(P)=P_{i i} \mu_{i i} / p_{i}+\frac{1}{2} \sum_{k, k \neq i} P_{i k} \mu_{i k} / p_{i} \text { for each } i
$$


and

$$
p_{i}^{\prime}=\frac{1}{2} p_{i}\left[(1+\sigma)+(1-\sigma) \mu_{i}(P) / \bar{\mu}\right] .
$$

For a polymorphic equilibrium $\hat{P}$, we have $\hat{p}_{i}{ }^{\prime}=\hat{p}_{i}$, and thus $\mu_{i}(\hat{P})=\bar{\mu}(\hat{P})$, from which follows

$$
\left.\hat{P}_{i j}=2 \hat{p}_{i} \hat{p}_{j}(1-\sigma) /\left(1-\frac{\sigma}{2}\right)\right]
$$

Hence

$$
\hat{I}_{i j}=\hat{I}=(1-\sigma) /\left(1-\frac{\sigma}{2}\right) .
$$

The equilibrium deficiency of heterozygotes relative to Hardy-Weinberg proportions is identical for all heterozygotes, does not depend on pollen production, and increases with an increasing rate $\sigma$ of ovule self-fertilisation.

Application of inequality (4): Inserting the parameters $\hat{\gamma}$ and $\hat{I}$ shows (provided $\mu_{a} \leqq \mu_{b}<\mu_{\text {het }}$ ) that the condition

$$
\frac{2(1-\sigma)}{\sigma} \frac{\mu_{\text {het }}-\mu_{b}}{\mu_{b}-\mu_{a}} \leqq r
$$

excludes the existence of a polymorphic equilibrium.

The reverse direction is necessary for its existence and holds if, for example $\mu_{a} \approx \mu_{b} \ll \mu_{\text {het }}$ and $\sigma$ sufficiently small. Fig. 1 illustrates the exact functional dependence.
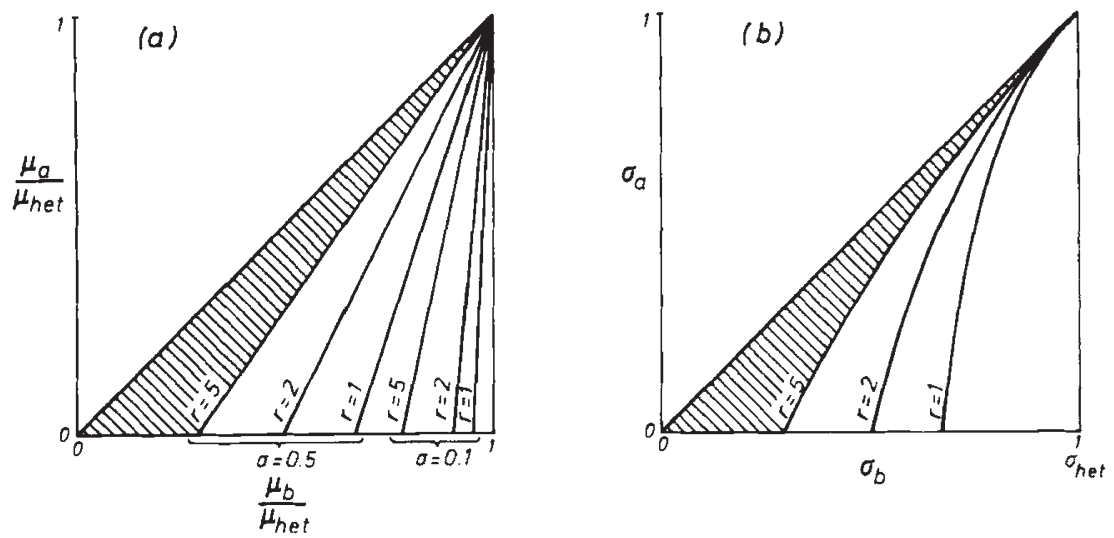

Illustration of parameter combinations which either exclude or allow the existence of polymorphic equilibria. Polymorphic equilibria can only occur if the parameters lie between the symmetry line $\left(\mu_{a}=\mu_{b}\right.$ for (a) or $\sigma_{a}=\sigma_{b}$ for (b)) and the $r$-line. They cannot occur for parameter combinations to the right of the $r$-line. As examples, those parameter regions which allow for the existence of polymorphic equilibria for the case $r=5$ (and additionally $\sigma=0.5$ for (a)) are hatched.

(a) Model of differential pollen production $\left(\mu_{a} \leqq \mu_{b}<\mu_{\text {het }}\right)$ and partial self-fertilisation ( $\sigma=$ 0.1 or $\sigma=0.5$ ).

(b) Differential rates of self-fertilisation $\left(\sigma_{a} \leqq \sigma_{b}<1\right)$ with $\sigma_{\text {het }}=1$. Analogously valid for positive assortative mating if the $\sigma$ 's are interpreted as assorting parameters. 
The diallelic case: For $r=1$ and provided $\mu_{11} \leqq \mu_{22}<\mu_{\text {het }}$, the equilibrium exclusion inequality reduces to $2(1-\sigma)\left(\mu_{12}-\mu_{22}\right) \leqq \sigma\left(\mu_{22}-\mu_{11}\right)$.

The reverse direction " $>$ " is proven to guarantee (under $\mu_{11} \leqq_{22}<\mu_{\text {het }}$ ) the protectedness of both alleles and the existence of a polymorphic equilibrium (Ziehe, 1982). Thus, the diallelic inequality turns out to be necessary and sufficient.

\section{(ii) Differing rates of self-fertilisation}

Model features for differential self-fertilisation in plant populations: Each individual produces a number of ovules which, when averaged with respect to the total ovule production of all individuals of the same genotype, amounts to $\phi$, a constant assumed to be independent of the particular genotype being considered. The same applies to the individual pollen contribution to the pollen cloud, i.e., the average contributions within the different genotypes are equal; an average proportion $\sigma_{i j}$ of ovules from individuals with genotype $A_{i} A_{j}$ is reserved for self-fertilization, the remaining ovules are fertilised at random by pollen from the pollen cloud.

Allelic selective asymmetry: The mean gametic contribution of individuals with genotype $A_{i} A_{j}$ to offspring zygotes results in

$\begin{array}{ll}\phi & \text { (ovule gametic contribution) } \\ +\sigma_{i j} \phi & \text { (pollen contribution via ovule self-fertilisation) } \\ +(1-\bar{\sigma}) \phi & \text { (pollen cross-fertilisation contribution) }\end{array}$

where $\bar{\sigma}=\sum_{j, i, i \leqq j} P_{i j} \cdot \sigma_{i j}$. Thus

$$
w_{i j}=w_{i j}(P)=\phi\left(2+\sigma_{i j}-\bar{\sigma}\right) .
$$

Under the restriction $\sigma_{11}=\cdots=\sigma_{m m}=\sigma_{a}, \sigma_{m+1, m+1}=\cdots=\sigma_{m+r, m+r}=\sigma_{b}$ and $\sigma_{i j}=\sigma_{\text {het }}$ for $i \neq j$ with $\sigma_{a} \leqq \sigma_{b}<\sigma_{\text {het }}, \gamma$ reads

$$
\gamma=\left(w_{\text {het }}-w_{b}\right) /\left(w_{\text {het }}-w_{a}\right)=\left(\sigma_{\text {het }}-\sigma_{b}\right) /\left(\sigma_{\text {het }}-\sigma_{a}\right)
$$

and is again frequency-independent, which implies in particular $\lambda=\hat{\gamma}$.

Heterozygote frequency deficiency in equilibrium: The transition equations read (c.f. e.g., Gregorius and Ross, 1981):

$$
P_{i j}=\sigma_{i j} P_{i j} / 2+\left[2-\sigma_{i}(P)-\sigma_{j}(P)\right] p_{i} p_{j} \text { for } i \neq j,
$$

where

$$
\sigma_{i}(P)=\sigma_{i i} P_{i i} / p_{i}+\frac{1}{2} \sum_{k, k \neq i} P_{i k} \sigma_{i k} / p_{i}
$$

and

$$
p_{i}^{\prime}=p_{i}+\frac{1}{2} p_{i}\left(\sigma_{i}(P)-\bar{\sigma}\right) .
$$

For a polymorphic equilibrium $\hat{P}$ with $\hat{p}_{i}^{\prime}=\hat{p}_{i}$, it immediately follows that $\sigma_{i}(\hat{P})=\bar{\sigma}(\hat{P})$ for all $i$. Thus

$$
\hat{P}_{i j}=2 \hat{p}_{i} \hat{p}_{i}\left[(1-\bar{\sigma}(\hat{P})) /\left(1-\frac{\sigma_{i j}}{2}\right)\right] \quad \text { for } i \neq j
$$

With the above restrictions on the $\sigma_{i j}$ 's, we obtain

$$
\hat{I}_{i j}=\hat{I}=(1-\bar{\sigma}(\hat{P})) /\left(1-\frac{\sigma_{\text {het }}}{2}\right) \text {. }
$$


Use of, for example, Ghai's (1974) method to obtain the corresponding frequency-independent solutions yields

$$
\hat{I}=\frac{m\left(1-\sigma_{a}\right)\left(\sigma_{\mathrm{het}}-\sigma_{a}\right)+r\left(1-\sigma_{b}\right)\left(\sigma_{\mathrm{het}}-\sigma_{b}\right)}{m\left(1-\frac{\sigma_{a}}{2}\right)\left(\sigma_{\mathrm{het}}-\sigma_{a}\right)+r\left(1-\frac{\sigma_{b}}{2}\right)\left(\sigma_{\mathrm{het}}-\sigma_{b}\right)+C},
$$

where

$$
C=\left(\frac{m+r}{2}-1\right)\left(\sigma_{\text {het }}-\sigma_{a}\right)\left(\sigma_{\text {het }}-\sigma_{b}\right) .
$$

Application of inequality (4): Inserting $\hat{\gamma}$ and $\hat{I}$ yields (provided $\sigma_{a} \leqq \sigma_{b}<$ $\left.\sigma_{\text {het }}\right)$ that the condition

$$
\frac{2\left(1-\sigma_{a}\right)}{\sigma_{\text {het }}} \frac{\left(\sigma_{\text {het }}-\sigma_{b}\right)}{\left(\sigma_{b}-\sigma_{a}\right)} \leqq r
$$

excludes the existence of a polymorphic equilibrium. The reverse direction holds if, for example, $\sigma_{a} \approx \sigma_{b} \ll \sigma_{\text {het }}$ and the ovule selfing rates all sufficiently small. Fig. 1(b) illustrates the different parameter regions for the special case $\sigma_{\text {het }}=1$.

The diallelic case: For $r=1$, the above inequality for the exclusion of equilibria reduces to (provided $\sigma_{11} \leqq \sigma_{22}<\sigma_{12}$ )

$$
2\left(1-\sigma_{11}\right)\left(\sigma_{12}-\sigma_{22}\right) \leqq \sigma_{12}\left(\sigma_{22}-\sigma_{11}\right) .
$$

The reverse direction " $>$ " guarantees not only the existence of a single polymorphic equilibrium but also of global convergence to it.

\section{(iii) Positive assortative mating}

Ghai (1974) presented a multiallelic model of positive assortative mating originating from the investigations of Karlin and Scudo (1969). The corresponding genotypic transition equations completely coincide with those above for differential self-fertilisation if the $\sigma_{i j}$ 's are replaced by assorting parameters. Hence all conclusions and the figure remain valid for positive assortative mating given the new interpretation of the parameters. For the diallelic case, an inequality equivalent to the above has been derived by Karlin (1969, p. 45). A detailed analysis concerning the evolutionary equivalence of positive assortative mating and differential self-fertilisation will be published elsewhere (Gregorius, 1984).

\section{Conclusions}

Models with frequency-independent heterozygotic fitness superiority and identical homozygotic fitnesses frequently show stable polymorphic equilibria. This is often valid even when the mating system components lead to a heterozygotic deficiency relative to Hardy-Weinberg proportions. As an example, consider Karlin and Farkash's (1978) multiallelic model of positive assortative mating. However, as shown in the preceding sections, identical homozygotic fitness behaviour which results in $\gamma=1$ is analytically an exceptional case. In particular for multiallelic model versions, even small 
deviations from the homozygotic identity in fitness can yield a definite exclusion of the existence of polymorphic equilibria. This fact restricts the general analytical importance of multiallelic symmetric models with a homozygotic excess and of their conclusions concerning polymorphic equilibria.

Suppose that the asymmetry measure $\gamma$ turns out to be frequencyindependent (as is the case for all examples in section 5) and that it is possible to derive upper and lower bounds for the panmictic index, which hold after a few generations at the latest. Then the way of proceeding used in section 3 can served to prove constant allelic fitness ranking after several generations and, thus, monotone allelic change and convergence of allelic frequencies.

Acknowledgements. This work was supported by a grant from the Deutsche Forschungsgemeinschaft, Bad Godesberg. The author wishes to thank G. Namkoong and H.-R. Gregorius along with anonymous referees for helpful discussions and comments, to E. Gillet for her help in preparing the manuscript and to B. Peter for her patience in carefully typing it.

\section{REFERENCES}

BROWN, A. H. D. 1979. Enzyme polymorphism in plant populations. Theoretical Population Biology, 15, 1-42.

GHAI, G. L. 1974. Analysis of some nonrandom mating models. Theoretical Population Biology, 6, 76-91.

GREGORIUS, H.-R. 1981. Realized genotypic fitnesses at equilibrium in the deterministic selection theory of a diallelic locus. Göttingen Research Notes in Forest Genetics, No. 4.

GREGORIUS, H.-R. 1982. The relationship between genic and genotypic fitnesses in diploid populations. Evolutionary Theory, 6, 143-162.

GREGORIUS, H.-R. 1984. Fractional fitnesses in exclusively sexually reproducing populations. Submitted for publication.

GREGORIUS. H.-R. AND ROSS, M. D. 1981. Selection in plant populations of effectively infinite size: I. Realized genotypic fitnesses. Mathematical Biosciences, 54, 291-307.

HAYMAN, B. I. 1953. Mixed selfing and random mating when homozygotes are at a disadvantage. Heredity, 7, 185-192.

JAIN, S. K. AND WORKMAN, P. L. 1967. Generalized F-statistics and the theory of inbreeding and selection. Nature, 214, 674-678.

KARLIN, S. 1969. Equilibrium behaviour of population genetic models with non-random mating. Gordon and Breach, New York.

KARLIN, S. AND FARKASH, S. 1978. Some multiallele partial assortative mating systems for polygamous species. Theoretical Population Biology, 14, 446-470.

KARLIN, S. AND SCUDO, F. M. 1969. Assortative mating based on phenotype. II. Two autosomal alleles without dominance. Genetics, 63, 499-510.

KIMURA, M. AND OHTA, T. 1971. Theoretical aspects of population genetics. Princeton University Press, Princeton, N.J.

LI. C. C. 1955. The stability of an equilibrium and the average of a population. American Naturalist, 89, 281-296.

ZIEHE, M. 1982. Zygotic genotypic frequencies under selection on female or male gamete production in partially self-fertilizing plant populations. Heredity, 49, 271-290. 